\title{
Beyond arbitrium: identification of a second communication system in Bacillus phage phi3T that may regulate host defense mechanisms
}

\author{
Charles Bernard $\mathbb{C}^{1,2} \cdot$ Yanyan $\mathrm{Li}^{2} \cdot$ Philippe Lopez $^{1} \cdot$ Eric Bapteste ${ }^{1}$
}

Received: 2 April 2020 / Revised: 17 September 2020 / Accepted: 24 September 2020 / Published online: 7 October 2020

(c) The Author(s) 2020. This article is published with open access

\begin{abstract}
The evolutionary stability of temperate bacteriophages at low abundance of susceptible bacterial hosts lies in the trade-off between the maximization of phage replication, performed by the host-destructive lytic cycle, and the protection of the phage-host collective, enacted by lysogeny. Upon Bacillus infection, Bacillus phages phi3T rely on the "arbitrium" quorum sensing (QS) system to communicate on their population density in order to orchestrate the lysis-to-lysogeny transition. At high phage densities, where there may be limited host cells to infect, lysogeny is induced to preserve chances of phage survival. Here, we report the presence of an additional, host-derived QS system in the phi3T genome, making it the first known virus with two communication systems. Specifically, this additional system, coined "Rap $\varphi$-Phr $\varphi$ ", is predicted to downregulate host defense mechanisms during the viral infection, but only upon stress or high abundance of Bacillus cells and at low density of population of the phi3T phages. Post-lysogenization, Rap $\varphi$-Phr $\varphi$ is also predicted to provide the lysogenized bacteria with an immediate fitness advantage: delaying the costly production of public goods while nonetheless benefiting from the public goods produced by other non-lysogenized Bacillus bacteria. The discovered "Rap $\varphi$-Phr $\varphi$ " QS system hence provides novel mechanistic insights into how phage communication systems could contribute to the phagehost evolutionary stability.
\end{abstract}

\section{Introduction}

Temperate bacteriophages are viruses that can infect their bacterial hosts either through the host-destructive lytic cycle or the non-destructive lysogenic cycle. During the latter stage, the phage replicates as part of the bacterial genome as a "prophage" and confers immunity to a second infection upon

These authors contributed equally: Philippe Lopez, Eric Bapteste

Supplementary information The online version of this article (https:// doi.org/10.1038/s41396-020-00795-9) contains supplementary material, which is available to authorized users.

Charles Bernard

charles.bernard@cri-paris.org

1 Institut de Systématique, Evolution, Biodiversité (ISYEB), Sorbonne Université, CNRS, Muséum National d'Histoire Naturelle, Campus Jussieu, Bâtiment A, 4eme et. Pièce 429, 75005 Paris, France

2 Unité Molécules de Communication et Adaptation des Microorganismes (MCAM), CNRS, Muséum National d'Histoire Naturelle, CP 54, 57 rue Cuvier, 75005 Paris, France the lysogenized bacterium $[1,2]$. Lysogeny, therefore, is a strategy that protects the phage-host collective [3].

Remarkably, Erez et al. observed that upon Bacillus subtilis infection, Bacillus phage phi3T (phi3T) relies on an endogenous quorum sensing (QS) system called "arbitrium" to orchestrate the lysis-to-lysogeny transition, when phages become abundant and the susceptible hosts left to infect are likely to be few [4].

Here, we report the discovery of a second communication system, termed "Rap $\varphi-P h r \varphi$ ", in the genome of phi3T, now the first known virus with two communication systems. $\operatorname{Rap} \varphi$-Phr $\varphi$ is the first known phage-encoded QS system predicted to regulate bacterial mechanisms, and in both a phage- and a host-density dependent manner.

\section{Results and discussion}

In addition to arbitrium, we report that Bacillus phage phi3T encodes a second QS system, coined "Rap $\varphi-P h r \varphi$ ", from the Rap-Phr family of Bacillus bacteria [5, 6] (Fig. 1a). The Rap $\varphi$ intracellular QS receptor (NCBI accession APD21157.1) is a 379 aa long protein that shows $48 \%$ 


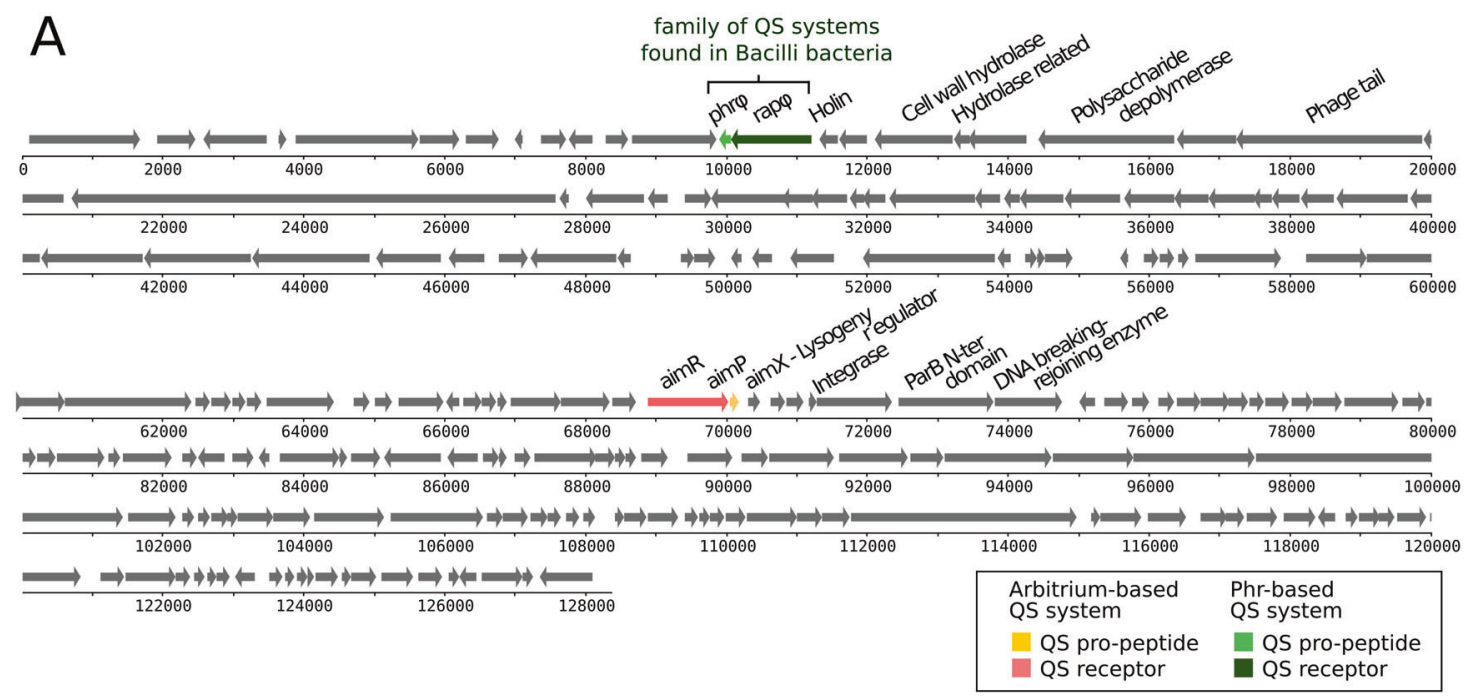

B
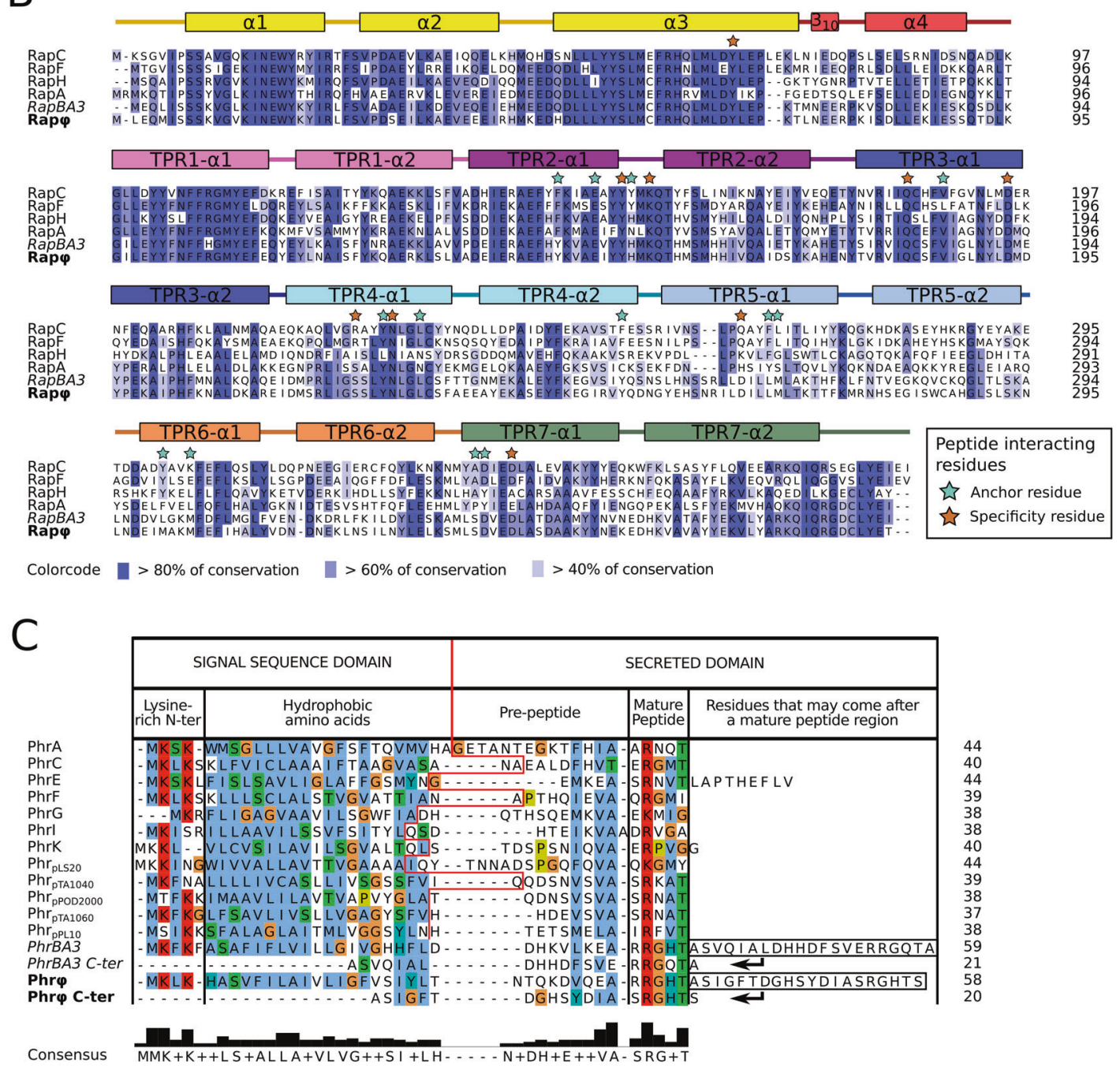

sequence identity over its entire length with RapC, a wellcharacterized member of the Rap protein family [7, 8] (Fig. 1b). Rap $\varphi$ harbors tetratricopeptide repeats (TPR)
(Pfam PF13181.6 and PF13424.6 HMM profiles), typically involved in the binding of QS peptides [9] (Fig. 1b). Phre (NCBI accession APD21156.1) is a 58aa long protein that 
Fig. 1 Characterization of the Rape-Phre QS system in Bacillus phage phi3T. a Position of the rap $\varphi$-phre and of the arbitrium (aimR$\operatorname{aim} P$ ) QS systems in the genome of phi3T. On this genomic map, arrows pointing towards the right correspond to genes on the "+" DNA strand whereas arrows pointing towards the left correspond to genes on the "-" strand. The position of each gene in the genome of phi3T is indicated by the horizontal axis (number of base pairs). The genes belonging to a QS system are in color. The aimX regulator of lysogeny is located downstream from aimP. The genes in the vicinity of each QS system are functionally annotated, whenever it was possible. b Multiple sequences alignment of the RapA, C, F and H proteins (B. subtilis str. 168) for which the structure has been resolved in Gallego del Sol and Marina [7] alongside RapBA3 (prophage of $B$. velezensis FZB42) and $\operatorname{Rap} \varphi$ (Bacillus phage phi3T). The N-terminal region of the Rap proteins is involved in the interaction with the ComA-P and/or Spo0F-P response regulators whereas the C-terminal region (TPR repeats) is involved in the interaction with the $\mathrm{Phr}_{\text {mature }}$ QS peptide. Residues are colored according to the "\% identity" colorcode. The residues that have been identified by Gallego del Sol and Marina as involved in the interaction with the QS peptide are annotated by stars, and brown stars specifically highlight the residues responsible for the specificity of Rap towards its cognate $\mathrm{Phr}_{\text {mature }}$ peptide. c. Multiple sequences alignment of the canonical Phr proteins considered in Pottathil et al [10] alongside the full PhrBA3 protein, the C-terminus of PhrBA3 that follows the first PhrBA3 $3_{\text {mature }}$ region (RRGHT), the full Phre protein, and the C-terminus of Phr $\varphi$ that follows the first $\operatorname{Phr} \varphi_{\text {mature }}$ region (RRGHT). Residues are colored according to the "Clustal" colorcode (see: http://www.jalview.org/ help/html/colourSchemes/clustal.html). The red line in the middle of the alignment separates the $\mathrm{N}$-terminal signal sequence domain from the C-terminal secreted domain of Phr proteins, as annotated by Pottathil et al. The sequences of the different QS pentapeptides are indicated by the "Mature Peptide" column.

exhibits all the characteristics of Rap cognate Phr pre-propeptides [10], both in terms of sequence composition (Fig. 1c) and genetic organization, with the $p h r$ gene directly downstream of rap, on the same DNA strand (Fig. 1a). Phr $\varphi$ is predicted to be secreted via the SEC-translocon by SignalP5.0 [11] (likelihood of 0.76) and harbors two characteristic regions that might be processed by extracellular proteases into the mature R-R-G-H-T and/or S-R-G-H-T QS signal pentapeptide(s) (Fig. 1c). Although non-canonical, the presence of two regions that are eventually matured into QS peptides was reported in many other functional Phr proteins [5]. The rap $\varphi$ phr $\varphi$ locus forms an operon, directly flanked downstream and upstream by intrinsic terminators (Table S1). The mapping of RNA-seq reads sequenced during the infection of Bacillus by phi3T [4] to the phi3T genome indicated that rap $\varphi$ and $p h r \varphi$ are expressed upon Bacillus infection (Fig. S1).

In the Bacillus genus, Rap-Phr systems are diverse, often strain specific and subject to horizontal transfers [5, 6]. We identified 2360 homologs of the Rap $\varphi$-Phr $\varphi$ QS systems in the complete genomes of Firmicutes available on the NCBI, including 333 predicted by Phaster [12] to belong to prophages, suggesting that rap-phr QS systems are widely used and horizontally transferred by phages (Table S2 and Fig. S2). Two of the QS systems carried by prophages (RapBA3-PhrBA3 in B. velezensis FZB42 and RapBL5-
PhrBL5 in B. licheniformis ATCC14580) have previously been functionally tested via a dedicated synthetic construct in B. subtilis strain PY79 (although it was then unknown that these systems belong to prophages) [5].

At low densities of the Bacillus subpopulation that either expresses the cloned rapBA3 or rapBA5 receptors, the Rap protein was experimentally demonstrated to strongly inhibit the Spo0F-P and ComA-P response regulators [5]. The SpoOF-P pathway leads to biofilm formation or sporulation $[13,14]$, shown to elicit the lytic cycle of certain bacteriophages $[15,16]$. The ComA-P pathway triggers competence and the production of antimicrobials [17] that might contribute to the defense against phages. As a Rap-Phr expressing Bacillus subpopulation grows, the secreted $\mathrm{Phr}_{\text {mature }}$ peptide accumulates in the medium. At high concentrations, $\mathrm{PhrBA} 3_{\text {mature }}$ and PhrBL5 $5_{\text {mature }}$ are internalized by the Opp permease and demonstrably sequester their cognate Rap receptor, which, in turn, alleviates the inhibition of ComA-P, Spo0F-P and their target pathways [5]. Therefore, there is evidence that Rap-Phr systems of prophages can delay, in Bacillus, defense mechanisms (antimicrobials, sporulation) and the production of public goods (biofilm molecules, antimicrobials) until the Bacillus subpopulation expressing the prophage QS system reaches a substantial cellular density (Figs. 2a and S3). Importantly, Rap $\varphi$-Phre is highly similar to RapBA3-PhrBA3. Rap $\varphi$ and RapBA3 yield $77 \%$ sequence identity at full mutual coverage and harbor the exact same residues that account for the QS peptide specificity (Fig. 1b). Consistently, Phr $\varphi$ presents a mature peptide region (R-R-G$\mathrm{H}-\mathrm{T}$ ), identical to the PhrBA3 3 mature QS peptide shown to inhibit RapBA3 [5] (Fig. 1c).

On these bases, we predict that Rap $\varphi$-Phr $\varphi$ is likely to be functional and that $\operatorname{Rap} \varphi$ can thus downregulate host defense mechanisms via Spo0F-P and ComA-P inhibition, enhancing the efficiency of the phi3T lytic cycle. Importantly, Rap $\varphi$ regulation of host processes is predicted to occur only at low concentrations of $\mathrm{Phr} \varphi_{\text {mature }}$ and when SpoOF is phosphorylated upon stress or ComA during the QS response of the entire, mixed population of Bacillus cells [13, 18, 19] (see refs. [20-23] for the potential advantages for a phage to inhibit the host's QS response) (Figs. 2 and S4). Hence, when defense pathways are triggered at high densities of Bacillus bacteria, phi3T phages might "disarm" their host only at low densities of phages, when the phage-host collective is not yet endangered. Meanwhile, the arbitrium system of phi3T ensures a fast-replication through the lytic cycle when phages are rare. When phages become abundant, it triggers a population-wide, host-protective lysogeny [4]. Particularly, if the quorum mediated by arbitrium were reached before that of $\operatorname{Phr} \varphi_{\text {mature }}$, the Rap $\varphi$ protein of phi3T prophages could delay the production of public goods in their freshly lysogenized hosts until the lysogenized subpopulation, likely afflicted by the previous lytic cycle, reaches a substantial density [19]. 


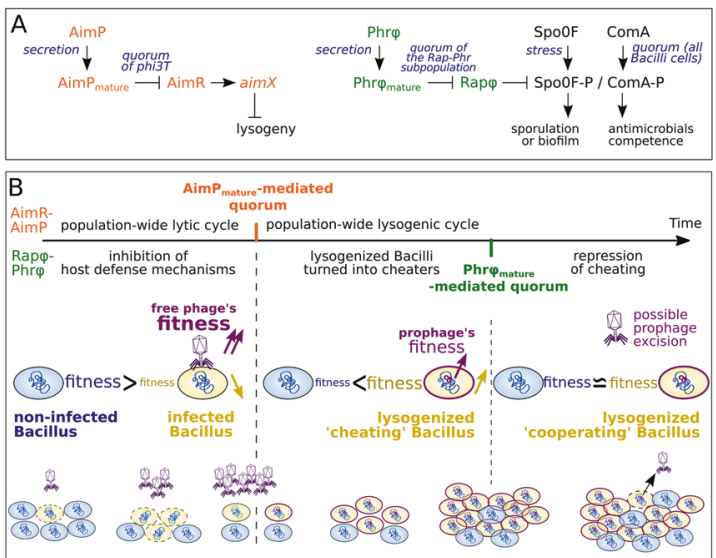

Fig. 2 Hypotheses on the mechanism of Rap $\varphi$-Phre, on its synergy with the arbitrium system, and on the evolutionary advantages for phi3T to own both systems. a Wiring diagrams of the characterized mechanism of the arbitrium system (left) and of the predicted mechanism of Rap $\varphi-P h r \varphi$ (right). Upon Bacillus infection, AimP and $\operatorname{Phr} \varphi$ are predicted to be exported in the medium by the SEC-translocon and cleaved by extracellular peptidases into mature QS peptides that accumulate in the medium as phi3T replicates. At low densities of phi3T, the unbound AimR QS receptor activates the expression of aimX encoding the negative regulator of lysogeny, which ensures a population-wide lytic cycle. At high densities of phi3T, AimP ${ }_{\text {mature }}$ reaches a threshold extracellular concentration and is consistently imported by neighboring cells via the Opp permease. Within infected Bacillus cells, AimP ${ }_{\text {mature }}$ inhibits AimR and alleviates lysogeny inhibition. A population-wide lysogeny is then triggered. At low densities of phi3T, the unbound Rap $\varphi$ QS receptor is predicted to inhibit the ComA-P and Spo0F-P response regulators of Bacillus (if the host is stressed or if the entire Bacillus population is at high density), which might downregulate host defense mechanisms. Either at high densities of phi3T or at high densities of the lysogenized subpopulation, $\mathrm{Phr}_{\text {mature }}$ is predicted to inhibit $\operatorname{Rap} \varphi$, which might reactivate the ComA-P and SpoOF-P pathways of the host. b A timeline is drawn on the top of the panel; the orange and the green vertical marks indicate when the quorums mediated by $\mathrm{AimP}_{\text {mature }}$ and by $\mathrm{Phr}_{\text {mature }}$ are proposed to intervene. The different mechanisms regulated by the arbitrium QS system over time are indicated on the topside of the timeline whereas the ones predicted to be regulated by $\operatorname{Rap} \varphi-\operatorname{Phr} \varphi$ are indicated on the bottom side. The predicted variations of density of the different subpopulations (phages phi3T, non-infected, infected and lysogenized Bacillus bacteria) over time are shown in the bottom of the panel. Their relative predicted fitness over time is shown in the middle of

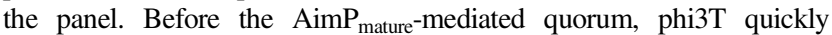
replicates at the expense of Bacillus during the lytic cycle. Meanwhile, Rap $\varphi$ might enhance the efficiency of the lytic cycle by downregulating host defense mechanism when Bacillus cells are abundant. When phages become abundant and when there may be limited host cells to infect, the AimP $P_{\text {mature }}$-mediated quorum triggers the host-protective lysogenic cycle and phi3T phages are internalized as prophages. The arbitrium QS system thus maximizes the time spent in the lytic cycle before it becomes a threat for the phage-host collective. After lysogeny, the Rap $\varphi$ protein of phi3T prophages might enhance the fitness of the prophage-host collective, by conferring an immediate fitness advantage on the freshly lysogenized subpopulation, likely afflicted by the previous lytic cycle. Indeed, whenever the entire Bacillus population would be at high densities and would transduce the signal for the production of public goods, the ComA-P and SpoOF-P inhibitions via $\operatorname{Rap} \varphi$ might provide the lysogenized subpopulation with the means to cheat (delay the costly production of biofilm molecules and antimicrobials) until this lysogenized subpopulation reaches again a substantial cellular density, as reflected by a threshold concentration of $\operatorname{Phr} \varphi_{\text {mature }}$.
Rap $\varphi$-Phr $\varphi$ could hence momentarily turn lysogenized Bacillus bacteria into cheaters, enhancing both the fitness of these cells and of the phi3T prophages. Interestingly, one prophage of B. velezensis SQR9 carried both the arbitrium and the Rap-Phr QS systems, as in phi3T (Fig. S2B), further supporting that owning both communication systems might be advantageous for a temperate bacteriophage.

\section{Data availability}

All data generated or analyzed during this study are included in this published article and its Supplementary information files. The genome of Bacillus phage phi3T can be accessed from the NCBI "Assembly" database with the "ASM260144v1" accession ID. The accession IDs for the protein sequences of Rap $\varphi$ and Phr $\varphi$ are "APD21157.1" and "APD21156.1", respectively.

Acknowledgements We would like to thank A. K. Watson for critical reading and discussion.

Author contributions C.B., Y.L., E.B., and P.L. conceived the study. C.B. performed the analyses. C.B., Y.L., and E.B. wrote the paper with input from all authors. All documents were edited and approved by all authors.

\section{Compliance with ethical standards}

Conflict of interest The authors declare that they have no conflict of interest.

Publisher's note Springer Nature remains neutral with regard to jurisdictional claims in published maps and institutional affiliations.

Open Access This article is licensed under a Creative Commons Attribution 4.0 International License, which permits use, sharing, adaptation, distribution and reproduction in any medium or format, as long as you give appropriate credit to the original author(s) and the source, provide a link to the Creative Commons license, and indicate if changes were made. The images or other third party material in this article are included in the article's Creative Commons license, unless indicated otherwise in a credit line to the material. If material is not included in the article's Creative Commons license and your intended use is not permitted by statutory regulation or exceeds the permitted use, you will need to obtain permission directly from the copyright holder. To view a copy of this license, visit http://creativecommons. org/licenses/by/4.0/.

\section{References}

1. Oppenheim AB, Kobiler O, Stavans J, Court DL, Adhya S. Switches in Bacteriophage Lambda Development. Annu Rev Genet. 2005;39:409-29.

2. Abedon ST. Bacteriophage secondary infection. Virol Sin. 2015; 30:3-10.

3. Wahl LM, Betti MI, Dick DW, Pattenden T, Puccini AJ. Evolutionary stability of the lysis-lysogeny decision: why be virulent? Evolution (N.Y). 2018;73:evo.13648. 
4. Erez Z, Steinberger-Levy I, Shamir M, Doron S, Stokar-Avihail A, Peleg Y, et al. Communication between viruses guides lysislysogeny decisions. Nature. 2017;541:488-93.

5. Even-Tov E, Omer Bendori S, Pollak S, Eldar A. Transient duplication-dependent divergence and horizontal transfer underlie the evolutionary dynamics of bacterial cell-cell signaling. PLoS Biol. 2016;14:e2000330.

6. Cardoso P, de F, Perchat S, Vilas-Boas LA, Lereclus D, VilasBôas GT. Diversity of the Rap-Phr quorum-sensing systems in the Bacillus cereus group. Curr Genet. 2019;65:1367-81.

7. Gallego del Sol F, Marina A. Structural basis of Rap phosphatase inhibition by Phr peptides. PLoS Biol. 2013;11:e1001511.

8. Reizer J, Reizer A, Perego M, Saier MH. Characterization of a family of bacterial response regulator aspartyl-phosphate (RAP) phosphatases. Micro Comp Genomics. 1997;2:103-11.

9. Do H, Kumaraswami M. Structural mechanisms of peptide recognition and allosteric modulation of gene regulation by the RRNPP family of quorum-sensing regulators. J Mol Biol. 2016; 428:2793-804.

10. Pottathil M, Lazazzera BA. The extracellular PHR peptide-Rap phosphatase signaling circuit of bacillus subtilis. Front Biosci. 2003;8:913.

11. Almagro Armenteros JJ, Tsirigos KD, Sønderby CK, Petersen TN, Winther O, Brunak S, et al. SignalP 5.0 improves signal peptide predictions using deep neural networks. Nat Biotechnol. 2019;37:420-3.

12. Arndt D, Grant JR, Marcu A, Sajed T, Pon A, Liang Y, et al. PHASTER: a better, faster version of the PHAST phage search tool. Nucleic Acids Res. 2016;44:W16.

13. Trach K, Burbulys D, Strauch M, Wu JJ, Dhillon N, Jonas R, et al. Control of the initiation of sporulation in Bacillus subtilis by a phosphorelay. Res Microbiol. 1991;142:815-23.

14. Molle V, Fujita M, Jensen ST, Eichenberger P, González-Pastor JE, Liu JS, et al. The Spo0A regulon of Bacillus subtilis. Mol Microbiol. 2003;50:1683-701.
15. Osburne MS, Sonenshein AL. Behavior of a temperate bacteriophage in differentiating cells of Bacillus subtilis. J Virol. 1976;19:26-35.

16. Meijer WJ, Castilla-Llorente V, Villar L, Murray H, Errington J, Salas M. Molecular basis for the exploitation of spore formation as survival mechanism by virulent phage $\varphi 29$. EMBO J. 2005; 24:3647-57.

17. Comella N, Grossman AD. Conservation of genes and processes controlled by the quorum response in bacteria: characterization of genes controlled by the quorum-sensing transcription factor ComA in Bacillus subtilis. Mol Microbiol. 2005;57:1159-74.

18. Bischofs IB, Hug JA, Liu AW, Wolf DM, Arkin AP. Complexity in bacterial cell-cell communication: quorum signal integration and subpopulation signaling in the Bacillus subtilis phosphorelay. Proc Natl Acad Sci USA. 2009;106:6459-64.

19. Kalamara M, Spacapan M, Mandic-Mulec I, Stanley-Wall NR. Social behaviours by Bacillus subtilis: quorum sensing, kin discrimination and beyond. Mol Microbiol. 2018;110:863.

20. Tan D, Hansen MF, de Carvalho LN, Røder HL, Burmølle M, Middelboe M, et al. High cell densities favor lysogeny: induction of an $\mathrm{H} 20$ prophage is repressed by quorum sensing and enhances biofilm formation in Vibrio anguillarum. ISME J. 2020;14: 1731-42.

21. Patterson AG, Jackson SA, Taylor C, Evans GB, Salmond GPC, Przybilski R, et al. Quorum Sensing Controls Adaptive Immunity through the Regulation of Multiple CRISPR-Cas Systems. Mol Cell. 2016;64:1102-8.

22. Høyland-Kroghsbo NM, Maerkedahl RB, Svenningsen SLO. A quorum-sensing-induced bacteriophage defense mechanism. MBio. 2013;4:e0362-12.

23. Høyland-Kroghsbo NM, Paczkowski J, Mukherjee S, Broniewski $\mathrm{J}$, Westra E, Bondy-Denomy J, et al. Quorum sensing controls the Pseudomonas aeruginosa CRISPR-Cas adaptive immune system. Proc Natl Acad Sci USA. 2017;114:131-5. 